\title{
VLBA multi-epoch water maser observations towards Cepheus A
}

José M. Torrelles ${ }^{1}$, Nimesh A. Patel ${ }^{2}$, José F. Gómez ${ }^{3}$, Paul T. P. Ho ${ }^{2}$, Luiz F. Rodríguez ${ }^{4}$, Guillem Anglada ${ }^{2,5}$, Guido Garay ${ }^{6}$, Lincoln Greenhill $^{2}$, Salvador Curiel ${ }^{7}$, Jorge Cantó ${ }^{7}$

${ }^{1}$ Institut d'Estudis Espacials de Catalunya (IEEC/CSIC) and Instituto de Ciencias del Espacio (CSIC), Edifici Nexus, C/ Gran Capità, 2-4, E-08034 Barcelona, Spain

${ }^{2}$ Harvard-Smithsonian Center for Astrophysics, 60 Garden Street, Cambridge, MA 02138

${ }^{3}$ Laboratorio de Astrofísica Espacial y Física Fundamental (INTA), Apdo. Correos 50727, E-28080 Madrid, Spain

${ }^{4}$ Instituto de Astronomía (UNAM), Apdo. Postal 72-3, (Xangari), 58089 Morelia, Michoacán, México

${ }^{5}$ Instituto de Astrofísica de Andalucía (CSIC), Ap. Correos 3004, E-18080 Granada, Spain

${ }^{6}$ Departamento de Astronomía, Universidad de Chile, Casilla 36-D, Santiago, Chile

${ }^{7}$ Instituto de Astronomía (UNAM), Apdo. Postal 70-264, DF 04510, México

Abstract. We have carried out and recently reported VLBA multiepoch water maser observations toward Cepheus A with an angular and velocity resolution of 0.5 mas and $0.2 \mathrm{~km} \mathrm{~s}^{-1}$, respectively. Some of the masers detected previously with the VLA (observations made with angular and velocity resolutions of 80 mas and $1.3 \mathrm{~km} \mathrm{~s}^{-1}$, respectively) are resolved now into linear/arcuate coherent "microstructures". These structures, while smaller than the VLA beam, are 6-200 times the size of the VLBA synthesized beam. The morphology and the observed proper motions found in these structures imply three different centers of star formation activity in a region of $\simeq 0$.' 3 radius $(\simeq 200 \mathrm{AU})$. The most remarkable result from these observations is the discovery of an arc of water masers which is perfectly fitted by a circle to one part in a thousand. This arc is expanding and suggests a spherical "puff" of ejected material ejected 33 years ago from a protostar located 600 mas south of the radio jet HW2. This spherical ejection represents a very strong challenge for theoretical studies of star formation.

\section{Introduction}

Associated with the thermal radio jet HW2 (Rodríguez et al. 1994) in the Cepheus A high-mass star forming region, there is one of the brightest clusters 
of water masers related with young stellar objects (YSOs), with flux densities up to $1000 \mathrm{Jy}$ (Lada et al. 1981, Cohen, Rowland, \& Blair 1984). Very Large Array (VLA) observations of the $1.3 \mathrm{~cm}$ continuum and water maser emission toward HW2, made with an angular resolution of 0.08 (Torrelles et al. 1996), show that the $1.3 \mathrm{~cm}$ continuum emission from this source is resolved into two maxima, as expected in a biconical ionized thermal jet, and is associated with a cluster of water masers. The spatial and kinematic distribution of these masers indicate that they trace a rotating and contracting circumstellar disk. In addition to HW2 and its associated water masers, four radio continuum $\mathrm{HW}$ objects (HW3b, HW3c, HW3d, and HW9; Hughes \& Wouterloot 1984, Hughes, Cohen, \& Garrington 1985), spread over $\sim 5^{\prime \prime}$ around HW2, and two spatial clusters of water masers associated with HW3b and HW3d were also detected (Torrelles et al. 1998).

In order to measure the proper motions of the water masers around HW2 we have carried out and recently reported (Torrelles et al. 2001a,b; hereafter Papers I and II, respectively) Very Long Baseline Array (VLBA) water maser measurements during three epochs (spread over two months). We summarize in this paper the main results obtained through these VLBA observations.

\section{Results}

The VLBA data obtained with an angular and velocity resolution of 0.5 mas and $0.2 \mathrm{~km} \mathrm{~s}^{-1}$, respectively, have revealed that some of the individual masers detected previously with the VLA are resolved now into remarkable linear/arcuate coherent "microstructures". In Figure 1 we show the water maser positions measured with the VLBA (dots) and VLA (crosses). The large box (dashed lines) indicates the region of the VLA water masers tracing the circumstellar disk with its major axis indicated by a solid line (Torrelles et al. 1996). The orientation and size (FWHM) of the radio jet associated with HW2 is shown by an ellipse, with its central position indicated by a star. The regions where the linear/arcuate water maser microstructures are detected with the VLBA (Papers I and II) are numbered from 1 to 5 in this figure.

Linear structures with sizes 3-25 mas (2-18 AU) and unresolved in the perpendicular direction are detected in regions 1,2 , and 3 (Fig. 1). The linear structure detected in region 1 , in its turn, is formed by even smaller $(0.4 \mathrm{AU})$ linear pieces. Lateral proper motions toward the northwest direction of $\sim 7 \mathrm{~km} \mathrm{~s}^{-1}$ are measured in the linear structure 1. The flattened appearance of these linear water maser microstructures suggests a shock nature, as expected from theory (Elitzur, Hollenbach, \& McKee 1992, Kaufmann \& Neufeld 1996). We have proposed (Paper I) that these three linear structures represent shock surfaces at the walls of a cavity created by the wind of HW2 in the circumstellar disk, interpreting the lateral proper motions of the water maser microstructure as due to the rotating motions of the disk.

An arcuate structure with proper motions of $\sim 30 \mathrm{~km} \mathrm{~s}^{-1}$ and size 35 mas (25 AU) is detected in region 4 (Fig. 1). We interpret this structure (Paper I) as a bow-shock excited by a wind of an energy source (other than HW2) located in region 4. This arcuate structure resembles the water maser "micro bow-shock" structure observed by Furuya et al. (2000) in the star-forming region S106. 


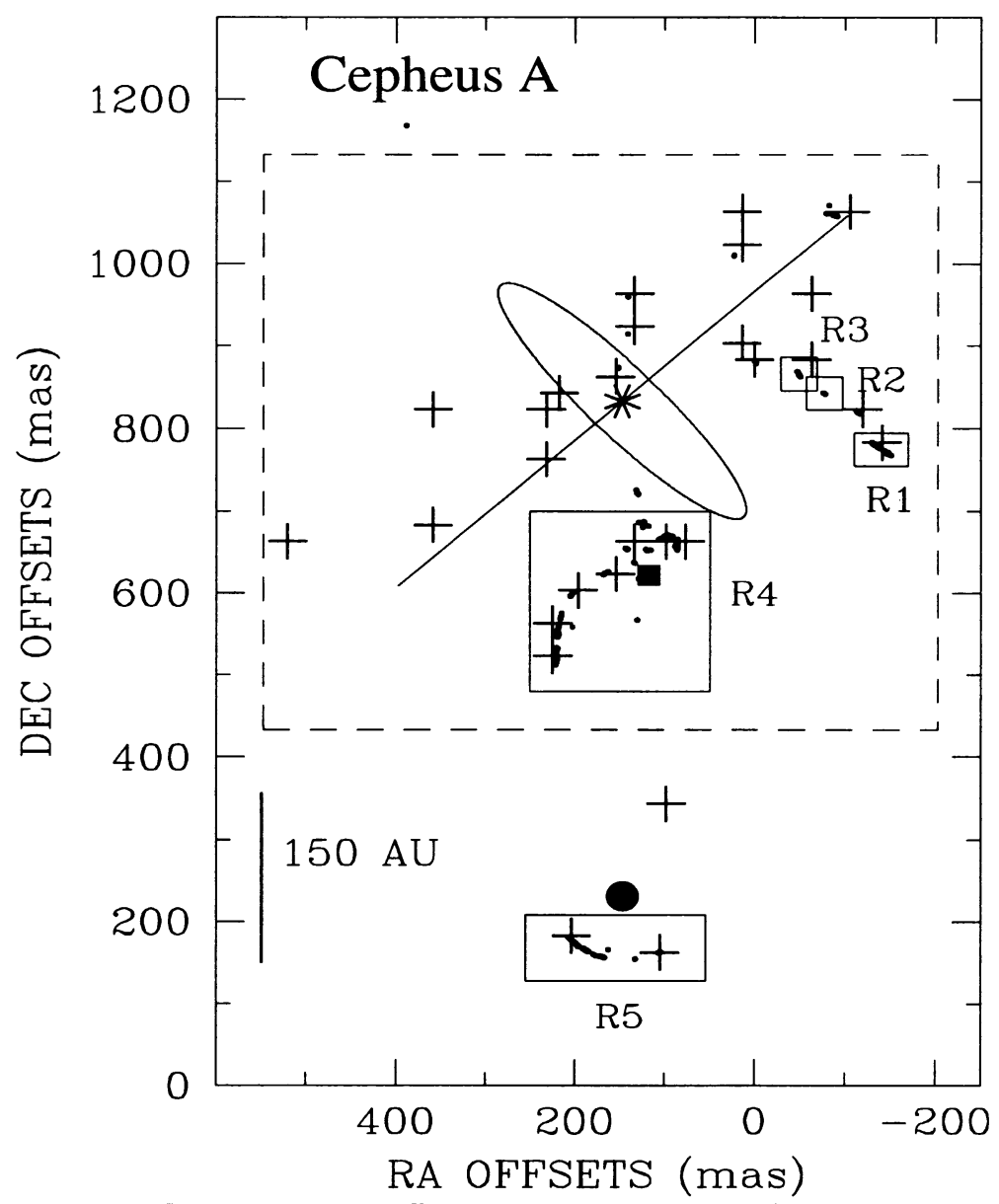

Figure 1. Water maser offset positions measured with the VLA (crosses) and VLBA (dots). The absolute coordinates of the reference position $(0,0)$ are $\alpha(\mathrm{J} 2000)=22^{h} 56^{m} 17^{s} .967, \delta(\mathrm{J} 2000)=62^{\circ} 01^{\prime}$ $48^{\prime \prime} 75$. The large box (dashed lines) shows the region of water masers measured with the VLA tracing the circumstellar disk. The solid line indicates the major axis of the disk. The ellipse shows the size (FWHM) and orientation of the radio jet HW2, with the position of its energy source indicated by a star. The small boxes labelled R 1 to $\mathrm{R} 5$ indicate the regions where the linear/arcuate water maser microstructures are detected with the VLBA. The filled square and circle mark, respectively, the positions where the energy sources of the bow-shock (region 4) and spherical "puff" of ejected material (region 5) are suspected to be located. These three energy sources, located inside a region of 600 mas $(400 \mathrm{AU})$ in size, could constitute a triple stellar system in a very early evolutive stage. (Figure adapted from Paper I). 
Finally, an arc-like structure with a length of $72 \mathrm{AU}$ is detected in region 5 (Paper II). This arc is extremely well fitted by a circle of $62 \mathrm{AU}$ radius to an accuracy of one part in a thousand. Moreover, this structure of masers is composed of very small linear structures (length $\simeq 0.4-1 \mathrm{AU}$ ), but unresolved in the perpendicular direction. These linear "building-blocks" are most likely highly flattened surfaces defining shock fronts, as expected from theoretical models (Elitzur, Hollenbach, \& McKee 1992, Kaufmann \& Neufeld 1996). Proper motions seen over a time span of two months demonstrate uniform expansion perpendicular to the arc at the rate of $9 \mathrm{~km} \mathrm{~s}^{-1}$. The high degree of symmetry exhibited both by the spatial structure and the proper motions led us (Paper II) to conclude that it represents the limb brightened parts of a spherical ejection driven by a protostar located at the center of the circle which fit the arc structure. This spherical "puff" of ejected material has a dynamical age of 33 years, suggesting that we may be seeing an explosive event originated during a very early evolutionary stage of a deeply embedded stellar object. We summarize that the observed spherical "puff" of water maser material strongly constrains the ejection process of the unknown central young stellar object to be spherically symmetric and short lived, representing a very strong challenge for theoretical studies of star formation.

\section{References}

Cohen, R. J., Rowland, P. R., \& Blair, M. M. 1984, MNRAS, 210, 425

Elitzur, M, Hollenbach, D. J., \& McKee, C. F. 1992, ApJ, 394, 221

Furuya, R. S., Kitamura, Y., Wootten, H. A., Claussen, M. J., Saito, M., Marvel, K. B., \& Kawabe, R. 2000, ApJ, 542, L135

Hughes, V. A., \& Wouterloot, J. G. A. 1984, ApJ, 276, 204

Hughes, V. A., Cohen, R. J., \& Garrington, S. 1995, MNRAS, 272, 469

Kaufmann, M. J., \& Neufeld, D. A. 1996, ApJ, 456, 250

Lada, C. J., Blitz, L., Reid, M. J., \& Moran, J. M. 1981, ApJ, 243, 769

Rodríguez, L. F., Garay, G., Curiel, S., Ramírez, S., Torrelles, J. M., Gómez, Y., \& Velázquez, A. 1994, ApJ, 430, L65

Torrelles, J. M., Gómez, J. F., Rodríguez, L. F., Curiel, S., Ho, P. T. P., \& Garay, G. 1996, ApJ, 457, L107

Torrelles, J. M., Gómez, J. F., Garay, G., Rodríguez, L. F., Curiel, S., Cohen, R. J., \& Ho, P. T. P. 1998, ApJ, 509, 262

Torrelles, J. M., Patel, N. A., Gómez, J. F., Ho, P. T. P., Rodríguez, L. F., Anglada, G., Garay, G., Greenhill, L., Curiel, S., \& Cantó, J. 2001a, ApJ, submitted [Paper I]

Torrelles, J. M., Patel, N. A., Gómez, J. F., Ho, P. T. P., Rodríguez, L. F., Anglada, G., Garay, G., Greenhill, L., Curiel, S., \& Cantó, J. 2001b, Nature, 411 [Paper II] 